\title{
COSTO-EFECTIVIDAD DEL PROGRAMA DEL CONTROL DEL NIÑO SANO EN EL PRIMER NIVEL DE ATENCIÓN
}

\section{COST-EFFECTIVENESS OF THE PROGRAM OF CONTROL OF THE HEALTHY CHILD IN THE FIRST LEVEL OF CARE}

\author{
Martha Villanueva R. (1,2), Enrique Villarreal R. (1), Emma Vargas D. (1), \\ Liliana Galicia R. (1), Lidia Martínez G. (1) \\ (1) Unidad de Investigación Epidemiológica y en Servicios de Salud Querétaro. \\ Instituto Mexicano del Seguro Social. Querétaro, México. \\ (2) Unidad de Medicina Familiar No. 16 "Arturo Guerrero Ortiz", \\ Instituto Mexicano del Seguro Social. Querétaro, México.
}

\begin{abstract}
Objective: To determine the cost-effectiveness of the control program of healthy children at the primary care level. Methodology: Children were divided into two groups, with and without allocation of milk, measured at 8, 10 and 12 months of age. We evaluated 200 children with weight for age and height for age. Total cost estimate included the unit cost and service utilization in Family Medicine, EMI, Preventive Medicine, Laboratory, Office and Pharmacy. Results: The results of height for age alternative were most cost effective in all three measurements in the group without milk allocation of milk. For $\$ 3,000$ invested in this option you get an effectiveness of $85.36 \%$ at 12 months, 96.67\% at 10 months and $98.05 \%$ at 8 months), while the effectiveness for the group without milk allocation was 69.53\% at 12 months, $82.46 \%$ at 10 months and $91.60 \%$ at 8 months. Conclusion: The most cost-effective control program for healthy children at the first level of care is the one withoyt allocation of milk.
\end{abstract}

Keywords: children, cost effectiveness, growth.

Este trabajo fue recibido el 7 de Marzo de 2011 y aceptado para ser publicado el 15 de Octubre de 2011.

\section{INTRODUCCIÓN}

La importancia de la atención primaria para la salud del niño según la Organización Mundial de la Salud (OMS), la Organización Panamericana de la Salud (OPS), y el Fondo de las Naciones Unidas para la Infancia (UNICEF) ha sido de creciente interés en los sistemas de salud con el objetivo de disminuir la morbilidad y mortalidad infantil $(1,2)$. Para lo cual en los sistemas salud se han creado programas integrados con acciones encaminadas a promover, prevenir, detectar y controlar factores de riesgo en este grupo de edad $(3,4)$.

El control del niño sano es una actividad de supervisión periódica tanto del estado de salud, como del crecimiento y desarrollo, desde que nace hasta los 6 años, realizada a través de acciones coordinadas de los servicios de Medicina Familiar, Enfermera Materno Infantil (EMI), Medicina Preventiva, Nutrición y Estomatología (5).

A través de la coordinación de estos servicios se rea- liza atención integral que incluye la detección precoz de problemas de salud, la prevención de accidentes, aplicación de inmunizaciones, evaluación del desarrollo psicomotor, la medición de peso y talla para evaluar su crecimiento y estado nutricional; la orientación a los padres sobre aspectos de la alimentación e higiene, así como promover la lactancia materna y el suministro de alimentación suplementaria a partir de los 6 meses de edad $(6,7)$.

Todas estas acciones médicas realizadas dentro de las instituciones de salud tienen un costo (8), por lo que la aplicación de la economía al área de la salud permite lograr las metas programadas con el mínimo de recursos disponibles para la mejora de la calidad de la atención en las intervenciones en salud (9). Dependiendo de lo que se quiera estudiar, se cuenta con una serie de análisis económicos para evaluar la eficiencia de los recursos asignados a la salud (10).

Los estudios de costos se utilizan para determinar el valor monetario de los servicios utilizados por los 
usuarios; en tanto que los estudios de efectividad se usan en la medición de los resultados obtenidos por las intervenciones de salud en situaciones concretas y reales (11).

En el caso de la evaluación de algunos aspectos del programa de control de niño sano los estudios económicos se han dirigido a costo y efectividad de manera separada $(12,13)$.

En los estudios de costos, se han evaluado los costos promedio y los costos totales del programa de atención (atención prenatal y control de niño sano) del servicio de EMI y su consulta se ha establecido en \$US13.4 (14).

En los estudios de costos, se han evaluado los costos promedio y los costos totales del programa de atención (atención prenatal y control de niño sano) del servicio de EMI y su consulta se ha establecido en US\$13.4 (14). En las consultas para el control del niño, no hay una cifra exacta que influya en su efectividad, existe una variación de 4 a 8 consultas (hasta los 18 meses de edad) y esto depende de cada organización de salud $(15,16)$.

Estudios de efectividad y costo-efectividad se han basado en la detección de enfermedades en niños escolares (mayores de 4 años) a partir de la talla (17).

El presente trabajo tiene como objetivo determinar la relación costo-efectividad del programa del control del niño sano en el primer nivel de atención.

\section{SUJETOS Y MÉTODO}

A través de un estudio de costo efectividad se analizaron expedientes electrónicos de niños que estuvieron en el programa del control del niño sano durante el primer año de vida en las Unidades de Medicina Familiar de la Delegación Querétaro de julio a agosto de 2010.

Se conformaron dos grupos de estudio acorde al total de la dotación de leche que se les otorgó: Grupo 1: sin dotación de leche; Grupo 2: con dotación de leche, medido a $\operatorname{los} 8,10$ y 12 meses.

Se estudiaron un total de 200 niños, empleando como marco muestral el listado de niños menores de 6 años usuarios de las Unidades de Medicina Familiar, seleccionándose aquellos niños que no padecían enfermedades crónicas y que fueron atendidos en la consulta de enfermeria materno infantil y Medicina Familiar en el primer año de vida y que estuvieron en control del niño sano. A partir de esta población se integraron a los grupos considerando el número de leche recibida.

Los indicadores peso para la edad y talla para la edad se construyeron a partir de las variables peso, talla, sexo y edad, los cuales se emplearon para determinar la efectividad, considerando como efectivo el porcentaje normal de niños con peso para la edad y talla para la edad. Para determinar el estado de crecimiento se utilizaron como referencia las tablas de crecimiento propuestas por la
OMS y adaptadas en la norma oficial Mexicana (18).

La estimación del costo total se calculó en pesos mexicanos multiplicando el costo unitario (19) por el promedio de la utilización del servicio en Medicina Familiar, de enfermeria materno infantil, Medicina Preventiva (vacunas aplicadas), Laboratorio (hematología química en sangre, examen completo de orina, grupo y $\mathrm{Rh}$, cultivos), Gabinete (RX) y Farmacia (dotación láctea).

Para el análisis costo-efectividad se establecieron diferentes escenarios para cada indicador de peso para la edad y talla para la edad: costo para la efectividad de cada uno de los dos grupos (alternativas), costo para la efectividad del $100 \%$, efectividad para el costo de cada uno de los dos grupos (alternativa), y efectividad por cada $\$ 3,000$. Todos medidos a los 8,10 y 12 meses. Como parte del análisis se realizaron promedios y porcentajes.

\section{RESULTADOS}

Se midió la efectividad a los 8, 10 y 12 meses de edad de dos alternativas, los que se les proporciono dotación láctea y los que no, donde el indicador peso para la edad, la efectividad más alta es del $70.73 \%$ que corresponde a aquellos que se les proporciono dotación láctea y medida a los 12 meses, para el indicado talla para la edad esta misma alternativa reporta el mayor nivel de efectividad con un $69.51 \%$ medido a los 12 meses. En la tabla 1 se presenta la efectividad de cada una de las alternativas por indicador.

El costo unitario más alto es el de medicina familiar (\$ 183); el uso más alto en las tres mediciones corresponde a la aplicación de vacunas. El costo total más alto corresponde a la alternativa con dotación en las tres mediciones siendo de $\$ 2.999 .25$ a los 12 meses, $\$ 2,489.17$ a los 10 meses y $\$ 2,183.46$ a los 8 meses.

La tabla 2 se presenta el costo unitario de cada servicio y el costo total por cada una de las alternativas y a $\operatorname{los} 8,10$ y 12 meses.

Para el indicador peso para la edad la alternativa con mejor relación costo-efectividad en las tres mediciones es en aquellos a los que no se les otorgó dotaciones lácteas, por $\$ 3,000$ invertidos en esta alternativa se obtiene una efectividad del $85 \%$ a los 12 meses, $97 \%$ a los 10 meses y $98 \%$ a los 8 meses, en tanto la efectividad para la alternativa con dotación es de $71 \%$ a los 12 meses, $75 \%$ a los 10 meses y $92 \%$ a los 8 meses. Las tablas 3 , 4 y 5 presentan el análisis costo efectividad utilizando como referencia cada una de las alternativas, así como el costo para efectividad del $100 \%$.

Para el indicador talla para la edad la alternativa con mejor relación costo efectividad en las tres mediciones es en aquellos a los que no se les otorgó dotaciones lácteas, por $\$ 3,000$ invertidos en esta alternativa se 
obtiene una efectividad del $85 \%$ a los 12 meses, $97 \%$ a los 10 meses y $98 \%$ a los 8 meses, en tanto la efectividad para la alternativa con dotación láctea es de $70 \%$ a los 12 meses, $82 \%$ a los 10 meses y $92 \%$ a los 8 meses. En las tablas 3,4 y 5 se presenta el análisis utilizando como referencia cada una de las alternativas, así como también el costo para efectividad del $100 \%$.

\section{DISCUSIÓN}

La evaluación de los programas de salud debe de ser una actividad permanente de los sistemas de salud en la búsqueda de identificar debilidades y áreas de oportunidad que permitan la mejor asignación y uso de los recursos disponibles. En este contexto la evaluación del programa del niño sano se presenta como una alternativa que en el caso particular del presente trabajo se aborda desde la perspectiva económica, específicamente la evaluación costo-efectividad, actividad íntimamente ligada con la toma de decisiones.

La composición de los grupos comparados se realizó a partir de la dotación láctea, lo cual permite una apreciación muy cercana a la realidad de los servicios de salud. Junto a ello el realizar la evaluación el primer año de vida permite una evaluación homogénea, y la adecuada utilización de los indicadores empleados podrían parecer los mas representativos para evaluar el crecimiento del lactante en el primer año de vida.
Los resultados de la evaluación de la efectividad medida a partir del peso para la edad y talla para la edad se podría decir que se encuentran en valores críticos, considerando que aproximadamente el $50 \%$ de la población se encuentra en valores normales, lo cual preocupa al identificar que se trata de una población con ingreso mensual permanente y con servicio de seguridad social, además de estar inscritos en el programa de control del niño sano.

Es verdad que la esencia del análisis costo efectividad es la comparación del costo y la efectividad en conjunto, no obstante es valido realizar la comparación de la efectividad y del costo de manera independiente.

Cuando se compara la efectividad entre los grupos, es decir el peso para la edad y la talla para la edad, lo que se aprecia son mejores resultados en el grupo de dotación láctea, esta es una evaluación eminentemente clínica y tiene valor, en consecuencia desde esta perspectiva la recomendación es ofrecer la dotación láctea.

De igual forma la comparación del costo entre los grupos reporta un costo más alto cuando se proporciona dotación láctea, aclarando que el monto reportado incluye la dotación láctea y el uso de los servicios de primer nivel de atención; desde esta perspectiva, la cual se basa exclusivamente en el costo, no se podría recomendar la dotación láctea.

Sin embargo en el actual contexto de los sistemas

\section{TABLA 1}

Evaluación de efectividad por tipo de indicador a los 8, 10 y 12 meses de edad.

\begin{tabular}{|c|c|c|c|c|c|c|}
\hline \multirow[t]{2}{*}{ Indicador } & \multicolumn{2}{|c|}{8 meses } & \multicolumn{2}{|c|}{$\begin{array}{l}\text { Efectividad } \\
10 \text { meses }\end{array}$} & \multicolumn{2}{|c|}{12 meses } \\
\hline & $\begin{array}{c}\text { Sin } \\
\text { dotación }\end{array}$ & $\begin{array}{c}\text { Con } \\
\text { dotación }\end{array}$ & $\begin{array}{c}\text { Sin } \\
\text { dotación }\end{array}$ & $\begin{array}{c}\text { Con } \\
\text { dotación }\end{array}$ & $\begin{array}{c}\text { Sin } \\
\text { dotación }\end{array}$ & $\begin{array}{c}\text { Con } \\
\text { dotación }\end{array}$ \\
\hline \multicolumn{7}{|l|}{ Peso/edad } \\
\hline Obesidad & 2.38 & 1.96 & 1.28 & 2.11 & 2.47 & 1.22 \\
\hline Sobrepeso & 16.67 & 9.8 & 10.26 & 6.32 & 9.88 & 4.88 \\
\hline Normal & 63.1 & 66.67 & 66.67 & 62.11 & 69.14 & 70.73 \\
\hline Desnutrición leve & 16.67 & 17.65 & 20.51 & 23.16 & 17.28 & 19.51 \\
\hline Desnutrición moderada & 1.19 & 3.92 & 1.28 & 6.32 & 1.23 & 3.66 \\
\hline Desnutrición grave & 0 & 0 & 0 & 0 & 0 & 0 \\
\hline \multicolumn{7}{|l|}{ Talla/edad } \\
\hline Alta & 2.38 & 0 & 2.56 & 1.05 & 1.23 & 1.22 \\
\hline Ligeramente alta & 15.48 & 9.8 & 12.82 & 5.26 & 14.81 & 6.1 \\
\hline Estatura normal & 58.33 & 66.67 & 62.82 & 68.42 & 65.43 & 69.51 \\
\hline Ligeramente baja & 22.62 & 18.63 & 17.95 & 23.16 & 14.81 & 20.73 \\
\hline Baja & 1.19 & 4.9 & 3.85 & 2.11 & 3.7 & 2.44 \\
\hline
\end{tabular}


de salud la tendencia se centra en realizar la evaluación conjunta del aspecto clínico y el aspecto económico, y en consecuencia se requiere del análisis costo efectividad para tomar la decisión.

Lo que revela el análisis del trabajo que aquí se pre- senta es una mejor relación costo efectividad cuando no se proporciona dotación láctea, por cada peso invertido se obtiene una mayor ganancia en salud, en consecuencia la recomendación se establece en torno a recomendar que el programa del control del niño sano no incluya la

\section{TABLA 2}

Costo unitario de los servicios utilizados por tipo de alternativas y costo total por alternativas a los 8,10 y 12 meses de edad.

\begin{tabular}{lccccccc}
\multicolumn{1}{c}{ Servicio } & \multicolumn{2}{c}{$\mathbf{8}$ meses } & \multicolumn{2}{c}{$\begin{array}{c}\text { costo total } \\
\text { 10 meses }\end{array}$} & \multicolumn{2}{c}{$\mathbf{1 2}$ meses } \\
& $\begin{array}{c}\text { Costo } \\
\text { Unitario }\end{array}$ & $\begin{array}{c}\text { Sin } \\
\text { dotación }\end{array}$ & $\begin{array}{c}\text { Con } \\
\text { dotación }\end{array}$ & $\begin{array}{c}\text { Sin } \\
\text { dotación }\end{array}$ & $\begin{array}{c}\text { Con } \\
\text { dotación }\end{array}$ & $\begin{array}{c}\text { Sin } \\
\text { dotación }\end{array}$ & dotación \\
Medicina Familiar & 183 & 292.8 & 435.54 & 333,06 & 481,29 & 435.54 & 587.43 \\
EMI & 142 & 512.62 & 597.82 & 623,38 & 729,88 & 744.08 & 874.72 \\
Vacunas & 74 & 973.84 & 1021.94 & 984,94 & 1028,60 & 1110.74 & 1172.16 \\
BH & 51 & 1.53 & 2.04 & 0 & 1.02 & 1.53 & 1.02 \\
QS & 51 & 0.51 & 1.53 & 0 & 0.51 & 1.02 & 0.51 \\
EGO & 51 & 0.51 & 1.53 & 0 & 0.51 & 1.02 & 1.53 \\
ES & 51 & 0 & 0.51 & 0 & 0 & 0 & 0 \\
Grupo-Rh & 51 & 0.51 & 1.53 & 0 & 0 & 0 & 0 \\
Tiempos & 51 & 1.02 & 1.02 & 0 & 0.51 & 0.51 & 0 \\
Cultivo & 51 & 0 & 0 & 0 & 0 & 1.02 & 0.51 \\
RX & 137 & 1.37 & 0 & 8.22 & 6,85 & 4.11 & 1.37 \\
Leche & 30 & 0 & 120 & 0 & 240 & 0 & 360 \\
costo total & & $1,784.71$ & $2,183.46$ & 1949,6 & 2489,17 & 2299.57 & 2999.25 \\
\hline
\end{tabular}

*El costo se mide en pesos mexicanos.

\section{TABLA 3}

Análisis costo-efectividad del indicador peso para la edad y talla para la edad a los 8 meses de edad.

\section{Indicador \\ Análisis \\ Alternativa}

Sin Dotación Con Dotación

Costo para efectividad de 0 dotaciones

Costo para efectividad con dotación

Peso / edad

Efectividad para costo de 0 dotaciones

Efectividad para costo con dotación

Costo para efectividad del $100 \%$

Efectividad por cada 3000 pesos

Costo para efectividad de 0 dotaciones

Costo para efectividad con dotación

Talla / edad Efectividad para costo de 0 dotaciones

Efectividad para costo con dotación

Costo para efectividad del $100 \%$

Efectividad por cada 3000 pesos

$\begin{array}{ccc}63 \% & \$ 1,785 & \$ 2,067 \\ 67 \% & \$ 1,886 & \$ 2,183 \\ \$ 1,785 & 63 \% & 54 \% \\ \$ 2,183 & 77 \% & 67 \% \\ & \$ 2,828 & \$ 3,275 \\ & 98 \% & 92 \% \\ 58 \% & \$ 1,785 & \$ 1,910 \\ 67 \% & \$ 2,040 & \$ 2,183 \\ \$ 1,785 & 58 \% & 54 \% \\ \$ 2,183 & 71 \% & 67 \% \\ & \$ 3,060 & \$ 3,275 \\ & 98 \% & 92 \%\end{array}$


dotación láctea.

No obstante se deberá reconocer que en este estudio no se consideraron otras variables que influyen en el crecimiento del niño, entre ellas el nivel socioeconómico de la población, porque a pesar que se trate de una población trabajadora con seguridad social, lo cual asegura un ingreso económico permanente, se deberá reconocer que al interior de ellos los sueldos percibidos presentan una amplia gama de variación, en función de ello tal vez se podría recomendar que en ulteriores estudios se realice la estratificación por nivel socioeconómico o en su defecto realizar el estudio en un estrato socioeconómico específico y analizar el comportamiento de los resultados.

En conclusión desde la perspectiva clínica la recomendación establece continuar con la dotación láctea, desde la perspectiva económica la recomendación se centra en retirar la dotación láctea, pero desde la perspectiva costo efectividad, en la cual se

\section{TABLA 4}

Análisis costo-efectividad del indicador peso para la edad y talla para la edad a los $\mathbf{1 0}$ meses de edad.

\begin{tabular}{|c|c|c|c|c|}
\hline \multicolumn{3}{|c|}{ Indicador } & \multicolumn{2}{|c|}{ Alternativa } \\
\hline & & & sin dotación & con dotación \\
\hline \multirow{5}{*}{ Peso / edad } & Costo para efectividad de 0 dotaciones & $67 \%$ & $\$ 1,950$ & $\$ 2,672$ \\
\hline & Costo para efectividad con dotación & $62 \%$ & $\$ 1,816$ & $\$ 2,489$ \\
\hline & Efectividad para costo de 0 dotaciones & $\$ 1,950$ & $67 \%$ & $49 \%$ \\
\hline & Efectividad para costo con dotación & $\$ 2,489$ & $85 \%$ & $62 \%$ \\
\hline & Costo para efectividad del $100 \%$ & & $\$ 2,924$ & $\$ 4,008$ \\
\hline \multirow{7}{*}{ Talla / edad } & Efectividad por cada 3000 pesos & & $97 \%$ & $75 \%$ \\
\hline & Costo para efectividad de 0 dotaciones & $63 \%$ & $\$ 1,950$ & $\$ 2,285$ \\
\hline & Costo para efectividad con dotación & $68 \%$ & $\$ 2,123$ & $\$ 2,489$ \\
\hline & Efectividad para costo de 0 dotaciones & $\$ 1,950$ & $63 \%$ & $54 \%$ \\
\hline & Efectividad para costo con dotación & $\$ 2,489$ & $80 \%$ & $68 \%$ \\
\hline & Costo para efectividad del $100 \%$ & & $\$ 3,103$ & $\$ 3,638$ \\
\hline & Efectividad por cada 3000 pesos & & $97 \%$ & $82 \%$ \\
\hline
\end{tabular}

\section{TABLA 5}

Análisis costo-efectividad del indicador peso para la edad y talla para la edad a los $\mathbf{1 2}$ meses de edad.

Indicador

Costo para efectividad de 0 dotaciones

Costo para efectividad con dotación

Peso / edad

Efectividad para costo de 0 dotaciones

Efectividad para costo con dotación

Costo para efectividad del $100 \%$

Efectividad por cada 3000 pesos

Costo para efectividad de 0 dotaciones

Costo para efectividad con dotación

Talla / edad
Efectividad para costo de 0 dotaciones

Efectividad para costo con dotación

Costo para efectividad del $100 \%$

Efectividad por cada 3000 pesos
Alternativa sin dotación con dotación

$\begin{array}{lll}69 \% & \$ 2,300 & \$ 2,932 \\ 71 \% & \$ 2,352 & \$ 2,999\end{array}$

$\$ 2,300$

$69 \%$

$54 \%$

\$ 2,999

$90 \%$

$71 \%$

$\$ 3,326$

$\$ 4,240$

$85 \%$

$71 \%$

$\begin{array}{lll}65 \% & \$ 2,300 & \$ 2,823 \\ 70 \% & \$ 2,443 & \$ 2,999\end{array}$

$\$ 2,254$

$65 \%$

$53 \%$

$\$ 2,761$

$85 \%$

$70 \%$

$\$ 3,515$

$\$ 4,315$

$85 \%$ 
realiza el análisis conjunto de los aspectos clínicos y de los económicos la recomendación señala retirar la dotación láctea del programa de control del niño sano.

\section{RESUMEN}

Objetivo: Determinar la relación costo-efectividad del programa del control del niño sano en el primer nivel de atención. Metodología: Estudio de costo-efectividad de niños. Se definieron dos grupos con y sin dotación de leche. Se midió a los 8, 10 y 12 meses de edad. Se evaluaron 200 niños con el indicador peso para la edad y talla para la edad, la estimación del costo total incluyó el costo unitario y la utilización del servicio en Medicina Familiar, EMI, Medicina Preventiva, Laboratorio, Gabinete y Farmacia. Resultados: Para el indicador talla para la edad la alternativa con mejor relación costo efectividad en las tres mediciones es sin dotación láctea, por $\$ 3,000$ invertidos en esta alternativa se obtiene una efectividad del $85.36 \%$ (12 meses), $96.67 \%$ (10 meses) y $98.05 \%$ (8 meses), en tanto la efectividad para el grupo con es de $69.53 \%$ (12 meses), $82.46 \%$ (10 meses) y $91.60 \%$ ( 8 meses).Conclusión: La mejor relación costo-efectividad del programa del control del niño sano en el primer nivel de atención es sin el otorgamiento de dotación láctea.

Palabras clave: niño, costo, efectividad, crecimiento.

Dirigir la correspondencia a:

Profesor

Enrique Villarreal Ríos

Zaragoza y 5 de febrero Col. Centro

Querétaro. Qro. CP 76 000. México

Tel: (442) 211-23-00 ext 51113

E-mail: felibree@infosel.net.mx

\section{BIBLIOGRAFÍA}

1. Walter W. Rosser. Research in Family/General Practice is Essential for Improving Health Globally. Ann Fam Med 2004;2:S2-S4.

2. Gutiérrez G, Guiscafré H, Verver H, Valdés J, Rivas E, Loyo E, Clavery M. Programa de atención a la salud del niño en México. Evaluación de la calidad de la atención integrada que se otorga en los centros de capacitación, Gac Méd Méx 2001;137(1): 21-9.

3. Muñiz-Hernández O. Programas Integrados de Salud (PREVENIMSS) Rev Med Inst Mex Seguro Soc 2006;44(Supl 1):S1-S2.

4. Programas Integrados de Salud. Proyecto Estratégico 2001-2003 Instituto Mexicano del Seguro Socia Rev Enferm IMSS 2005;13(1):41-5

5. Trejo y Pérez J. Guía clínica para la vigilancia de la nutrición, el crecimiento y el desarrollo del niño menor de cinco años Rev Med IMSS
2003;41(Supl1):S47-S58.

6. González-Unzaga M. Martínez-Andrade G. Maya J. Ramos-Hernández R. Turnbull B. Flores S. Duque X. Flores-Huerta S. Martínez-Salgado H. Crecimiento en peso y longitud de los niños menores de dos en el IMSS Rev Med Inst Mex Seguro Soc 2007;45(1):37-46.

7. Muñiz-Hernández O. Programas Integrados de Salud (PREVENIMSS) Rev Med Inst Mex Seguro Soc 2006;44(Supl 1):1-2.

8. Baly GA, Toledo ME, Rodríguez JF. La economía de la salud, la eficiencia y el costo de oportunidad. Rev Cubana Med Gen Integr 2001;17(4):395-8.

9. Rodríguez-Ledezma MA, Constantino-Casas, García-Contreras F, Garduño-Espinoza J. Conocimientos de los médicos sobre la economía de la salud Rev Med Inst Mex Seguro Soc 2007;45 (4):343-352.

10. Taboadela C. Economía de la Salud. Actualización en Medicina del Trabajo. Asociart SA ART 2006;3(4):1-16.

11. Lisboa BC. Investigación en costo-efectividad. Boletín Escuela Med Pontificia Universidad Católica de Chile 1995;24:150-3.

12. Torre P. los sucedáneos de la leche materna en la seguridad social mexicana durante 1990. Salud Publica Mex 1993; 35: 700-8.

13. Mamani R. Efectividad del programa educativo "niño sano y bien nutrido para mejorar los conocimientos, actitudes y prácticas de seguridad nutricional aplicado a madres con niños menores de 1 año del centro de salud Moyopampa, Chosica-Lima. Rev Científica Enfermería. Recién 2008. p 43-50.

14. Villareal-Rios E, et al. Variación del costo unitario en una institución de seguridad social en queretaro, Mexico. Rev Salud Pública, 2006; 8(2):153-62.

15. Colomer Revuelta J. Grupo PrevInfad. ¿Cuántos controles son necesarios en el programa de actividades preventivas? En: Colomer Revuelta J. Curso de Actualización Pediatría 2004. Madrid: AEPAp eds; 2004: p 57-60.

16. Garrido Torrecilla FJ. Programa de Control del niño Sano, Evidencia científica, Ped Rur Ext 2007; 37(350):27-33.

17. Fayter D y col. Efectividad y costo-efectividad de los programas de rastreo de problemas de la talla en niños escolares. Arch Dis Child 2008;93;278-84.

18. Norma oficial Mexicana. NOM-031-SSA2-. Para la atención a la salud del niño. 1999.

19. El sistema de salud perfil de uso y costo en primer nivel de atención. Unidad de investigación epidemiológica y en servicios de salud Querétaro. 2007. 